\title{
The effects of genital myiasis on the diversity of the vaginal microbiota in female Bactrian camels
}

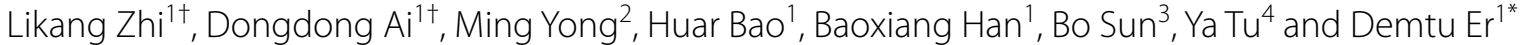

\begin{abstract}
Background: Genital myasis is one of the most important diseases that affects the reproductive organs of Bactrian camels in which can cause serious mechanical damage to the vaginal tissue. The accumulation of bacteria in the vagina of female camels can affect their health and reproductive ability. The effect of this damage is commonly manifested in the vaginal flora and vaginal mucosal immune system. Therefore, this investigation is a study of the diversity of the vaginal flora and the differences between healthy Bactrian camels and those suffering from genital myiasis.
\end{abstract}

Results: Vaginal microbiota samples were collected from two groups of female Bactrian camels of the same age. An Illumina MiSeq was used to sequence the 16S rRNA V3-V4 hypervariable sequence in the samples. The results showed that the vaginal microflora of the infected camels had a significantly greater operational taxonomic unit (OTU) value. According to the assessment of the alpha diversity index and the vaginal $\mathrm{pH}$, the diversity index of the infected camel flora was higher than that of the normal camel flora, and the vaginal pH was lower than that of the normal camels $(p<0.01)$. There were no significant differences between the two groups in the abundance of dominant genera in the Bactrian camel vagina $(P>0.05)$, indicating that the certain stability is maintained.

Conclusions: Overall, this comparison revealed the differences and similarities between the vaginal microbiota of Bactrian camels in various health statues. In addition, these data provide a reference point for understanding the types of bacteria that cause genital myiasis affecting the healthy development of Bactrian camels.

Keywords: Bacteriome, Bactrian camels, Genital myiasis, Vaginal flora diversity, 165 rRNA

\section{Background}

The Bactrian camel is one of the unique domestic animals in China. It mainly lives in the hot and arid regions of the Gobi and desert area in northwestern China. It is known as the "boat of the desert" [1]. For a long time, the development of the Bactrian camel breeding industry has been

\footnotetext{
*Correspondence: eedmt@imau.edu.cn

†Likang Zhi and Dongdong Ai contributed equally to this work.

${ }^{1}$ College of Veterinary Medicine, Key Laboratory of Clinical Diagnosis

and Treatment Technology in Animal Disease, Ministry of Agriculture

and Rural Affairs, Inner Mongolia Agricultural University, Hohhot 010018,

P.R. China

Full list of author information is available at the end of the article
}

hampered by genital myiasis, which has been linked to serious economic losses to local herders.

Genital myiasis of Bactrian camels is a serious parasitic disease. Larvae of Wohlfahrtia magnifica [2] parasitize the perineal and vaginal mucosal and cutaneous regions of Bactrian camels where cause traumatic lesions [3]. Genital myiasis has a distinct seasonality and occurs in May-September of each summer and autumn $[4,5]$. Clinical symptoms in diseased camels are the result of severe mechanical damage to the affected tissue and mucosal sites, causing harmful effects, such as local inflammation, anxiety and anorexia [5-7]. Through longterm experimental observation, we found that the diseased camel's vaginal wound is exposed to the external 
environment but is rarely infected or purulent. When the larvae of Wohlfahrtia magnifica are detached from the host, $94.5 \%$ of the diseased camel wounds spontaneously recover [2]. In addition, other important elements affect the vaginal microenvironment.

The vaginal mucosa in healthy animals is colonized by an equilibrated and dynamic population of aerobic, facultative anaerobic and obligate anaerobic microbes [8]. The vaginal flora constitutes a natural barrier formed on the surface of the vaginal mucosa, but some factors can disturb the balance in its composition [9]. Disruption of the vaginal microbiota equilibrium promotes infectious clinical syndromes with diverse symptoms, such as vaginal discharge, irritation, pruritus, and vulvar burning [10]. The formation of a reciprocal symbiotic relationship between the vaginal flora and the host is an important factor in maintaining the stability of the vaginal microenvironment. It is also a relevant component of the multifaceted resistance of female mammals to pathogen invasion. This has a major impact on the health and disease of the host organism. The vaginal microbiota has importance in preserving vaginal health and defending the host against disease [11]. Thus, the vaginal microbiome can indicate the health or disease status of the female camel and whether there are changes according to the treatment of any existing vaginal-related diseases [12-16].

At present, research on vaginal microbiology is mainly focused on humans. There are minimal data on the vaginal microflora in livestock species as well as its potential role in animal vaginal mucosal immunology. Research on the vaginal microbiology of Bactrian camels has not yet been conducted. Therefore, in this study, we completed the first high-throughput sequencing analysis of the vaginal flora of Bactrian camels. By comparing the diversity of the vaginal microbiota and differences between the diseased and healthy group in the same herd, the effects of environmental and nutritional factors on the vaginal bacterial community were eliminated, and an analysis was performed to determine the immune-related differences in microbiome composition.

Through this study, a new understanding of the vaginal mucosal immune mechanism of the Alxa Bactrian camel and its mechanism and resistance to vaginal myiasis caused by the larvae of Wohlfahrtia magnifica was obtained, which lays the foundation for future research and provides a new idea for the prevention and treatment of vaginal myiasis in the Alxa Bactrian camel.

\section{Results \\ Vaginal pH}

The results showed that the vaginal $\mathrm{pH}$ of the healthy group of Bactrian camels ranged from 7.47 to 8.23, with an average of $7.85 \pm 0.13$. The vaginal $\mathrm{pH}$ of the diseased group was significantly lower $(P<0.01)$ in the range of $7.18-7.61$ with an average of $7.41 \pm 0.11$.

\section{Sequencing information}

After optimization of quality control and chimaera removal, a total of 1,644,139 reads were obtained for all 23 samples, resulting in an average of 71,484 reads per sample (Table 1). Samples from the diseased group were taken and yielded a total of 744,455 reads, with an average of $77,446 \pm 11,214$ reads per sample. The healthy group samples yielded a total of 899,684 reads with an average of $69,206 \pm 11,047$ reads per sample. The results showed that there was no statistically significant difference in the number of optimized sequences between the two groups of samples $(P>0.05)$.

\section{Alpha and beta diversity analyses}

The sequences obtained above were subjected to merging and operational taxonomic unit (OTU) division based on $97 \%$ sequence similarity. Additionally, the OTUs with abundance values lower than $0.001 \%$ of the total sample sequencing amount were removed [17]. A total of 1845 OTUs were detected, with an average of 1689 in each sample. Moreover, 1267 OTUs were detected in the diseased group. In addition, the normal vaginal flora for each was maintained with 1111 OTUs shared between various vaginal environments (Fig. 1).

Alpha diversity was measured and observed using OTU, Chao1, ACE, Simpson (the Simpson diversity index is derived based on the assumption that two individuals are randomly selected in an infinite community and based on the probability that they belong to the same species) and Shannon diversity indices (the Shannon diversity index is a way to measure the diversity of

Table 1 Sequence and alpha diversity statistics of the 16S rRNA gene sequences for bacterial populations in the vagina of diseased and healthy camels

\begin{tabular}{lllllll}
\hline Group & Sample No & Average of sequence No & Simpson index & Chao1 & ACE & Shannon index \\
\hline Diseased & 10 & $77,446 \pm 11,214$ & $0.94 \pm 0.04$ & $495.04 \pm 230.85$ & $497.33 \pm 228.58$ & $5.55 \pm 1.05$ \\
Healthy & 13 & $69,206 \pm 11,047$ & $0.94 \pm 0.03$ & $361.65 \pm 147.45$ & $364.26 \pm 148.16$ & $5.07 \pm 0.59$ \\
$P$ & -- & 0.28 & 0.98 & 0.11 & 0.11 & 0.17 \\
\hline
\end{tabular}




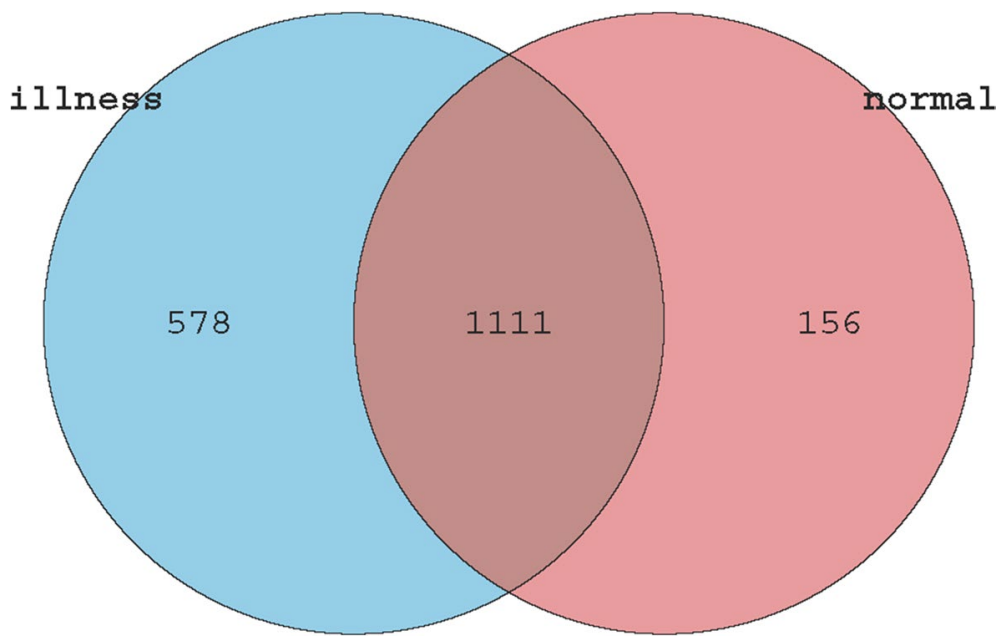

Fig. 1 Total OTU Venn diagram

species in a community). The conclusive analysis results are presented in Table 1 . No significant differences existed in alpha diversity between the healthy samples of female Bactrian camels and those of ill camels, according to the vaginal bacteria observed by the OTU, Chaol, ACE, Simpson and Shannon's diversity indices $(p>0.05)$. However, the vagina of diseased female Bactrian camels had a significantly greater number of OTUs than the normal Bactrian camel vagina, with increased richness as measured by Chaol and ACE and greater diversity as measured by Shannon's diversity index and the Simpson index, all of which are presented in Table 1.

Beta-diversity was also analyzed to examine differences in microbial communities between samples. Using an OTU-centric approach PCoA matrices were employed using weighted and unweighted UniFrac distance matrices to compare the phylogenetic divergence among the OTU between samples from ill camels and healthy camel vaginal samples (Fig. 2). The results showed that the subsets of healthy camel vaginal samples were more closely clustered in the weighted and unweighted UniFrac distance matrix. In addition, (Analysis of similarities) ANOSIM showed that there was a significant difference between the vaginal samples of diseased camels and healthy camels $(P=0.033)$. Statistical analysis showed that the difference between the groups was significantly greater than that within groups $(R=0.1483)$, and the grouping effect was evidently good.

\section{Taxonomic composition analysis}

According to the results of OTU classification and classification status identification, the dominant vaginal flora and average relative abundance of Bactrian camels in the healthy group and the diseased group were, respectively, identified at the phylum level: Firmicutes $(39.33 \% \pm 16.228 .34 \% \pm 19.39)$; Proteobacteria $\quad(28.48 \% \pm 15.0635 .23 \% \pm 14.18)$; $\quad$ Fusobacteria $\quad(16.12 \% \pm 11.3216 .86 \% \pm 13.55)$; $\quad$ Bacteroidetes $(6.04 \% \pm 3.58 .95 \% \pm 3.6)$; and Actinobacteria $(7.5 \% \pm 3.07 \% \pm 6.17 \% \pm 4.21)$. The average relative abundance of unallocated taxa was $(1.45 \% \pm 2.81 \% \pm 1.72 \% \pm 2.81)$. The relative abundance of the dominant vaginal flora of the Bactrian camels was not significantly different between the healthy group and the diseased group $(P>0.05)$.

At the genus level, the dominant bacteria and their average relative abundance identified from the vaginas of Bactrian camels in the normal group and the group of ill female camels were Campylobacter $(9.58 \% \pm 7.03 \% \pm 9.9 \% \pm 10.05)$; Ochrobactrum $\quad(5.76 \% \pm 6.037 .56 \% \pm 5.99) ;$ Fusobacterium (6.42\% $\pm 5.596 .6 \% \pm 5.84) ; \quad$ GW-34 $\quad(5.96 \% \pm 7.535 .58 \% \pm$ 12.15); Porphyromonas $\quad(4.08 \% \pm 3.574 .96 \% \pm 3.98)$; Facklamia $\quad(6.08 \% \pm 4.582 .33 \% \pm 1.15)$; $\quad$ Sediminibacterium $(1.45 \% \pm 1.65 \% \pm 2.48 \% \pm 2.56)$; Helcococcus $(1.25 \% \pm$ 2.32.38\% \pm 4.05$) ; \quad$ Peptoniphilus $\quad(1.04 \% \pm 1.052 .11 \% \pm 1.85)$; $1-68 \quad(1.59 \% \pm 2.220 .93 \% \pm 1.56) ; \quad$ Clostridium $\quad(1.22 \% \pm$ $1.071 .23 \% \pm 1.3)$; Acinetobacter $(1.18 \% \pm 1.281 \% \pm 0.78)$; Fusibacter $\quad(1.29 \% \pm 1.680 .44 \% \pm 0.62)$; Sphingomonas $(0.68 \% \pm 0.631 .09 \% \pm 0.86)$; and ph2 $(0.9 \% \pm 0.830 .53 \% \pm 0.98)$. The relative abundance of the dominant vaginal flora of the Bactrian camels was not significantly different between the healthy and diseased group $(P>0.05)$.

The genera of vaginal microorganisms that could not be identified or placed in defined categories in either the healthy or diseased Bactrian camels and their average relative abundances were as follows: family Leptotrichiaceae $(9.58 \% \pm 7.03 \% \pm 9.9 \% \pm 10.05)$, family Aerococcaceae $(7.78 \% \pm 5.182 .71 \% \pm 4.2)$, family Carnobacteriaceae $6.81 \% \pm 7.080 .3 \% \pm 0.62$ ), family Xanthomonadaceae 

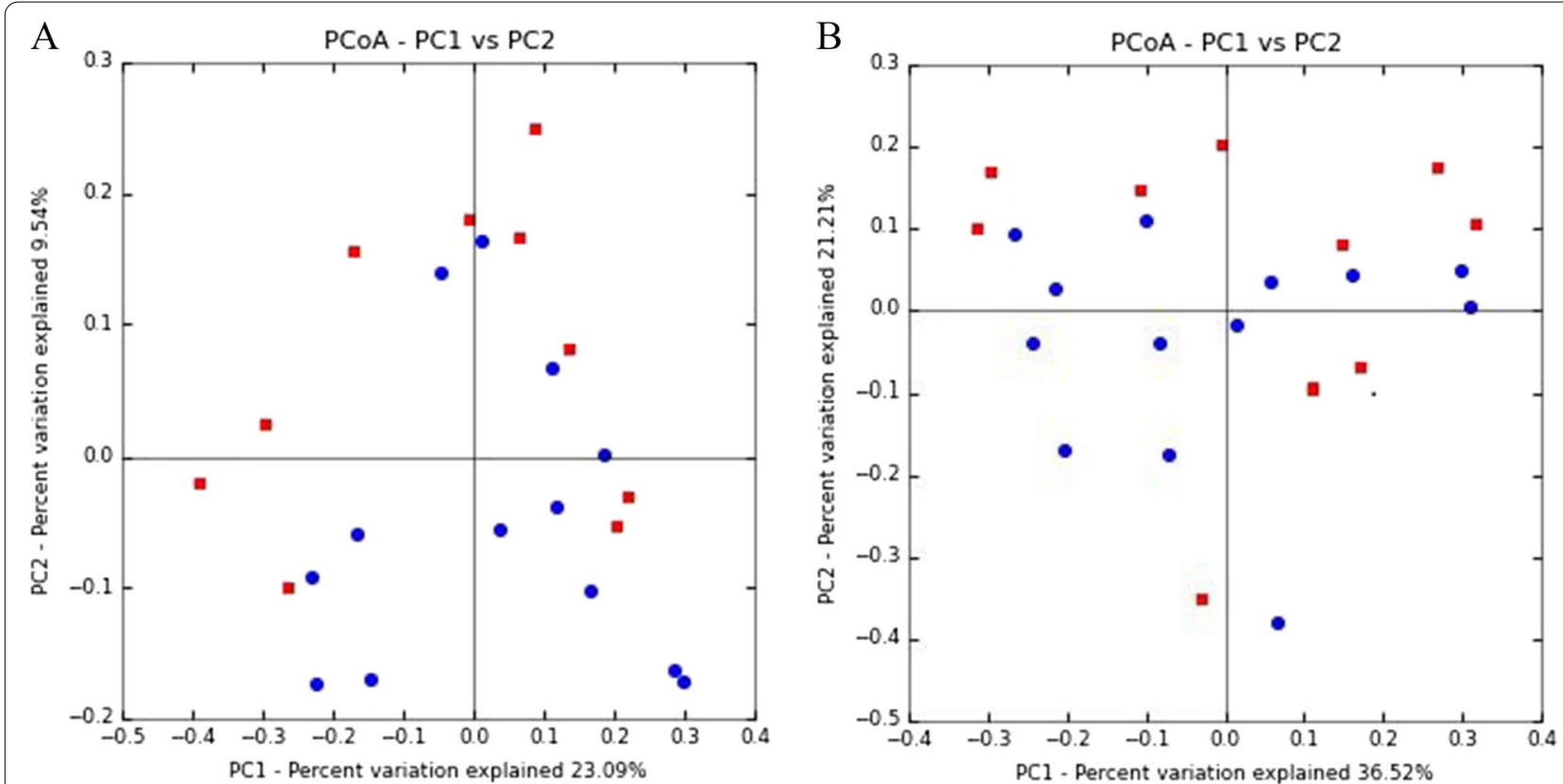

Fig. 2 Principal coordinate analysis of vaginal samples from diseased female camels and healthy female camels using UniFrac unweighted (A) and weighted (B) metrics. Vaginal samples from diseased female camels $(n=10)$ are represented by red squares, and vaginal samples from healthy camels $(n=13)$ are represented by blue circles

$(1.13 \% \pm 1.092 .97 \% \pm 2.96)$, family Pseudomonadaceae $(1.9 \% \pm 2.520 .9 \% \pm 0.75)$, family Tissierellaceae $(1.39 \% \pm 1.491 .12 \% \pm 1.75)$, family Ruminococcaceae $(0.35 \% \pm 0.391 .69 \% \pm 3.14)$, family Enterobacteriaceae $(0.47 \% \pm 0.781 .39 \% \pm 2.04)$, and family Comamonadaceae $(0.35 \% \pm 0.391 .02 \% \pm 0.78)$.

The visualization tool GraPhlAn [18] was used to build a hierarchical tree of the composition of the sample population at each classification level (Fig. 3).Using mothur software, the statistical algorithm Metastats (http://metastats.cbcb.umd. edu/) [19] was used. We were able to determine the overall classification level of all classification units in the sample population. The difference of sequence quantity (i.e., absolute abundance) between each taxon at the phylum and genus levels was analyzed and compared (pairwise). We found that there were 4 classification units with significant differences in taxon levels (Fig. 4), namely, SR1 $(p=0.030 q=0.120$ ), Planctomycetes $(p=0.030 q=0.120)$, Gemmatimonadetes $(p=0.041 q=0.120)$, and Elusimicrobia $(p=0.048 q=0.120)$. There were 51 taxonomies with significant differences in levels (Fig. 5), mainly Anaerostipes $(p=0.001 q=0.005)$, Caldilinea $(p=0.001 q=0.005)$, Edwardsiella $(p=0.001 q=0.005)$, Lactobacillus ( $p=0.027 q=0.064)$, etc.

\section{Discussion}

The implications and analysis of this study has provided further information the vaginal microecosystem of Bactrian camels. Relevant studies have proven that the combination of the microflora related to the human body can affect human immunity and provide the first line of defence against opportunistic pathogen colonization [20]. The importance of microbial metabolism to the host immune system can be revealed by characterizing the composition and function of individual microbial species and complex microbial communities [21]. This study is based on a basic research analysis. By comparing the differences in the structure and diversity of healthy camels and diseased camels, we were able to analyze the role of the vaginal microecosystem of Bactrian camels in their immunity and recovery stages after vaginal myiasis infection. Additional understanding of these stages may provide a new approach for the prevention and treatment of genital myiasis of Bactrian camels, which will result in positive advances for the clinical treatment of genital myiasis.

In this study, the bacterial phyla with the highest abundance identified in the two groups of Bactrian camel vaginal samples were Firmicutes, Proteobacteria, Fusobacterium and Bacteroides. These phyla are representative of the most common phyla found in many environments, especially in host-microbiome relationships. Previous studies have shown that the proportions and relative abundances of these taxa are related to changes in host physiology [22]. Therefore, when we performed ANOSIM on the samples, we found that even if there were differences among several individuals 


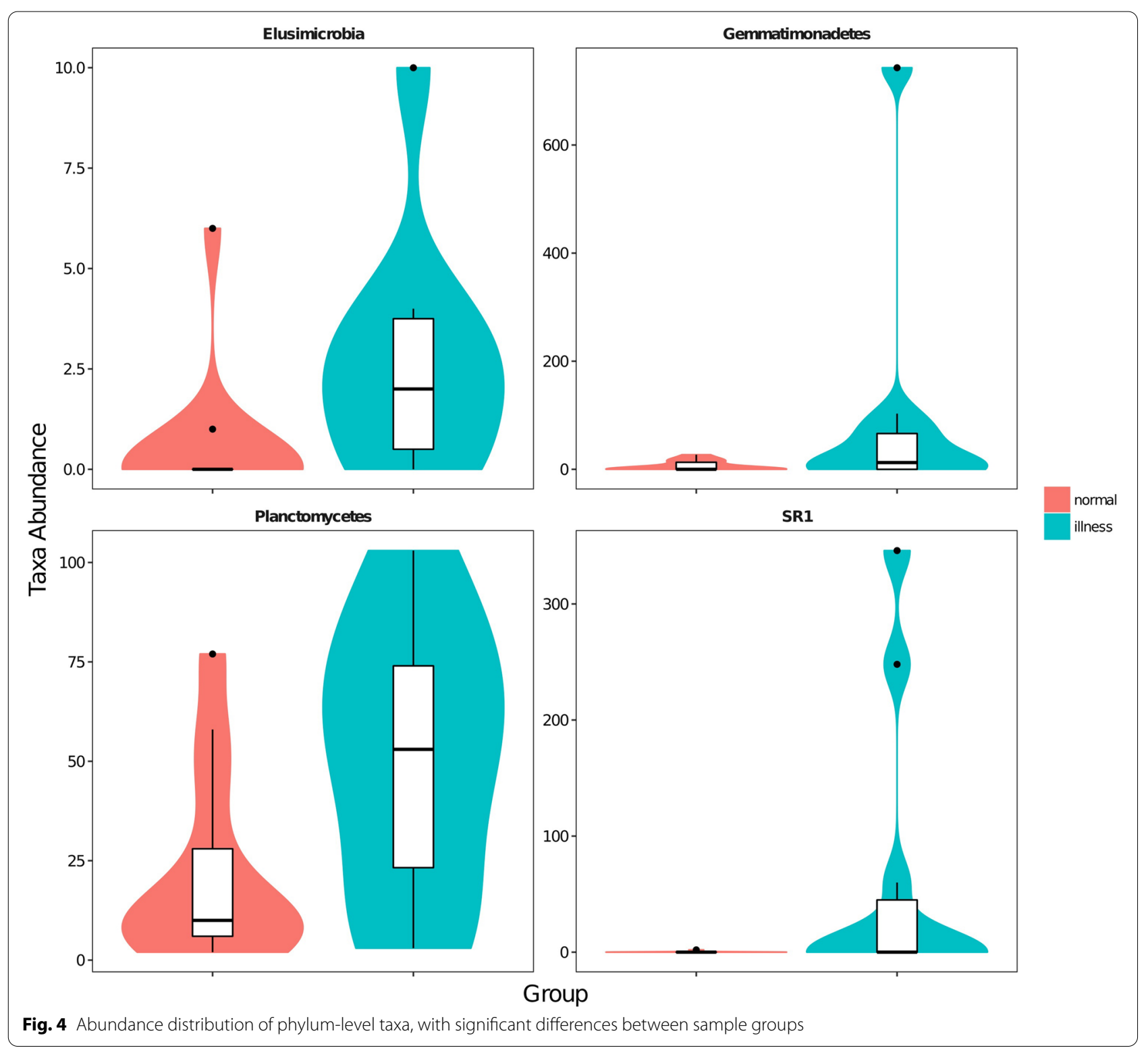

no significant differences in the overall structure of the vaginal flora, indicating that the vaginal microecology of Bactrian camels had certain stability. In addition, immunomodulatory symbionts induce specific self-targeted responses that indirectly regulate immune responses to surrounding microorganisms [23]. Thus, the key role of microbial flora in maintaining homeostasis in the vaginal environment has been demonstrated [24], and the vaginal-associated microbiota may significantly affect the vaginal mucosal regulation of Bactrian camels. For example, the flora on the vaginal mucosa reduces the colonization of pathogenic bacteria by competing for living space and nutrients together with the production of short-chain fatty acids, bacteriocins, and reactive oxygen species to inhibit or kill pathogenic bacteria [25]. When the larvae of Wohlfahrtia magnifica invade the vagina of Bactrian camels, the external environment of microorganisms enters the vagina of the diseased camel, and vaginal pathogenic bacteria stimulate the mucosal immune system. For example, inflammation strengthens the clearance of pathogenic bacteria and reduces the possibility of pyogenic disease. Therefore, the vaginal mucosal immune system is able to identify beneficial microorganisms and harmful microorganisms, and pathogens are eliminated by the body's clearance immune response, while commensal bacteria remain safe [26]. 


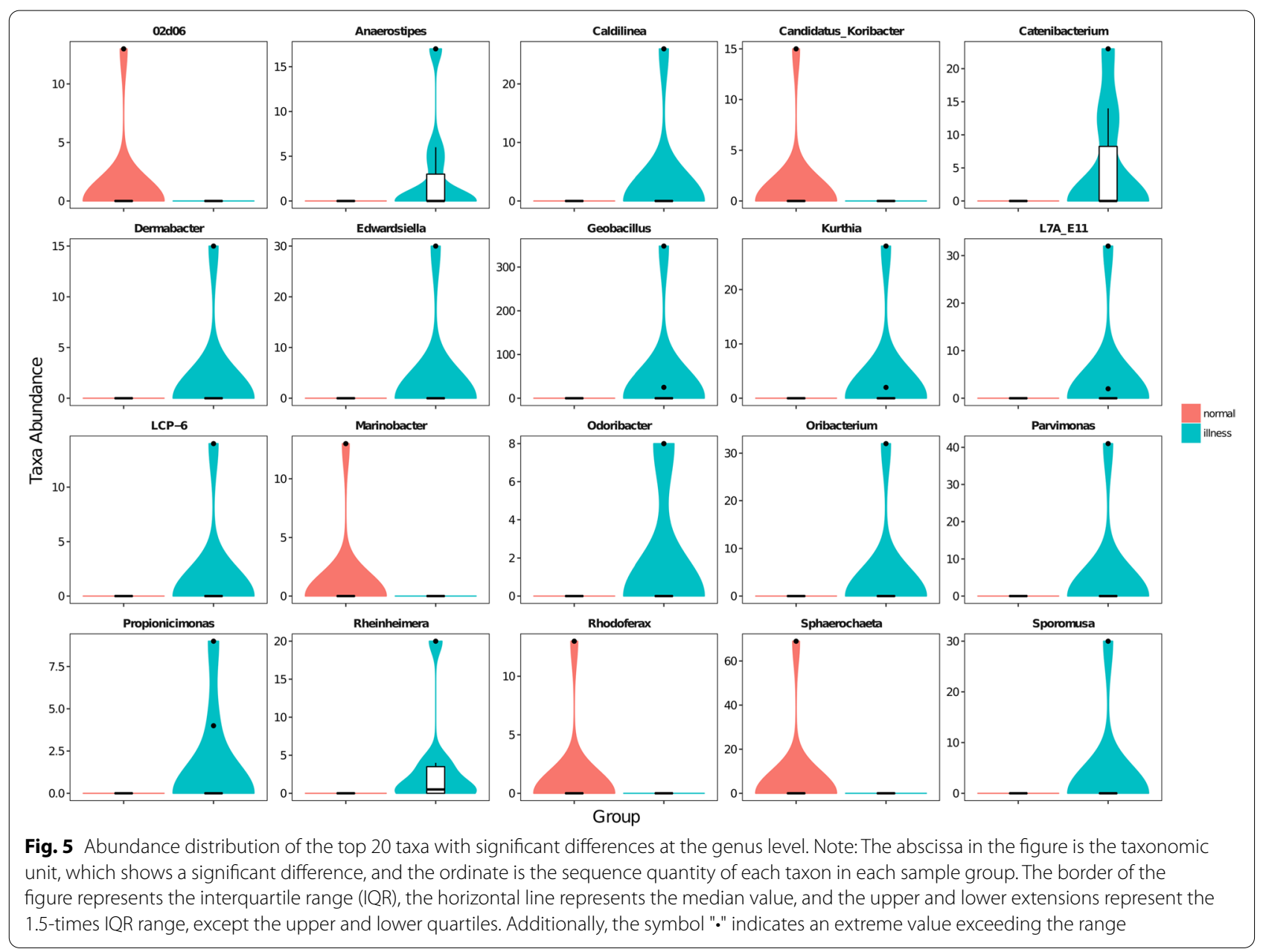

The chemical nature of the vaginal mucosal niche drives the composition of symbiotic microorganisms with unknown microbial roles and host factors that lead to differences in its microecological composition and strain levels [27]. The acid-producing genera of Bactrian vaginal flora in the illness group were significantly enriched, such as Lactobacillus, Edwardsiella, Oribacterium, Parvimonas, Propionicimonas, and Sporomusa. This result is consistent with the low $\mathrm{pH}$ found, suggesting that maintaining a low vaginal $\mathrm{pH}$ prevents the colonization of pathogenic microorganisms and has a positive impact on the body resistance to pathogen invasion [28-33]. Studies have shown that the production of lactic acid and other antimicrobial metabolites by the vaginal microflora prevents endogenous opportunistic bacterial proliferation and immunomodulation $[34,35]$. Lactobacillus is an important probiotic in the reproductive tract of female animals and can convert lactose and other sugars into lactic acid, which can prevent infection and reduce the risk of inflammation
[36-38]. Lactobacillus also plays a role in accelerating the healing of tissue wounds [39].

\section{Conclusions}

Through high-throughput sequencing of the vaginal flora of Bactrian camels, the diversity of the vaginal microbiota was revealed, and it has been demonstrated that genital myasis affects its composition. This study lay the basis for future research and proposed a new idea for the prevention and treatment of camel vaginal myiasis.

\section{Methods}

\section{Experimental design and sampling}

All female Bactrian camels involved in this study were part of a Bactrian camel herd registered with the College of Veterinary Medicine Inner Mongolia Agricultural University. All experimental procedures were approved by the Animal Protection and Use Committee of Inner Mongolia Agricultural University and strictly followed animal welfare and ethical guidelines [40]. 
According to the farming standards, all 23 Bactrian camels, including 10 suffering from genital myiasis and 13 healthy camels, were mature female, 8-years old animals. In addition, the camels studied were free-ranged and had no supplementary feeding except drinking water; additional findings were as follows: there was no history of vaginal drug release within one year; no oestrus or pregnancy for one month; and no antibiotics or antifungal drugs were used systematically within one month.

Routine sterile operations were used before each sampling and strictly followed. In addition, the procedural steps strictly ensured an aseptic opening of the female camel's vagina and swabbing 5 times along the vaginal wall to collect the vaginal secretions. Then, the swabs were quickly placed in sterile $5 \mathrm{ml}$ cryotubes. Finally, the samples were labelled and quickly stored in liquid nitrogen or at $-80{ }^{\circ} \mathrm{C}$ and used to extract the $16 \mathrm{~S}$ rRNA gene. Shortly afterwards, the $\mathrm{pH}$ of each sample was measured using an UltraBasic $\mathrm{pH}$ metre (Denver Instruments, Arvada, CO, United States).

\section{Bacterial DNA isolation}

The thawed sample was centrifuged at $10,000 \mathrm{rpm}$ for 10 min to collect bacterial cells, and the supernatant was discarded. The total DNA of the sample was extracted using a vaginal swab genomic DNA kit (Qiagen QIAamp DNA Mini Kit), and the specific steps were performed according to the instructions. The DNA was extracted and stored at $-20{ }^{\circ} \mathrm{C}$. The extracted DNA quality was evaluated by $0.8 \%$ agarose gel electrophoresis, and the DNA was quantified with an ultraviolet spectrophotometer.

\section{Sequencing of $16 \mathrm{~S}$ rRNA}

In combination with the fluorescence quantification results, each sample was mixed in a corresponding ratio according to the sequencing amount requirement of each sample. The processed samples were sent to Beijing WEISHENGTAI Co., Ltd. for paired-end $2 \times 300 \mathrm{bp}$ sequencing with the Illumina HiSeq 2000 platform.

\section{Statistical analysis}

Basic statistical analysis was performed using SPSS statistics 20.0 statistical analysis software. Two pairs of comparisons of the measured data were performed, in accordance with the normal distribution. Two independent samples tests were performed, and $P<0.05$ was therefore considered statistically significant.

Beta diversity was analyzed to examine differences in microbial communities between samples. Using an OTU-centric approach, (Principal Co-ordinates Analysis)PCoA matrices were employed using weighted and unweighted UniFrac distance matrices to compare the phylogenetic divergence among the OTUs between diseased camel samples and healthy camel vaginal samples.
The visualization tool GraPhlAn [18] was used to build a hierarchical tree of the composition of the sample population at each classification level. Each classification unit was distinguished by different colours, and their distribution in abundance was also reflected by the node size.

Using mothur software, the statistical algorithm Metastats (http://metastats.cbcb.umd.edu/)was used. We were able to determine the overall classification level of all classification units in the sample population. The difference of sequence quantity (i.e., absolute abundance) between each taxon at the phylum and genus levels was analyzed and compared (pairwise).

\section{Abbreviations}

OUT: Operational Taxonomic Unit; ANOSIM: Analysis of similarities; PCOA: Principal Co-ordinates Analysis.

\section{Supplementary Information}

The online version contains supplementary material available at https://doi. org/10.1186/s12917-022-03189-5.

Additional file 1.

Additional file 2.

Additional file 3.

Additional file 4.

Additional file 5.

Additional file 6.

Additional file 7.

\section{Acknowledgements}

The authors would like to acknowledge and thank the technical support of Shunyao Jiang. We thank all partners and laboratory members for their kind help.

\section{Arrive guidelines declaration}

During the whole experiment, we contact with bactrian camels during the taking samples, only. The sampling process does not cause any damage to the animals. We confirm that the study was conducted in accordance with the Arrival Guidelines.

\section{Authors' contributions}

DE conceived and designed the study, revised the manuscript and funded it LZ, DA and BH performed the experiments, analyzed the results, and drafted the manuscript. $\mathrm{HB}$ and BS assisted in experimental design, data interpretation and manuscript preparation. YT and MY participated in the sample collection, assisted in gene sequencing experiments and contributed to revise the manuscript. All authors read and approved the final manuscript.

\section{Funding}

This study was supported by The National Natural Science Foundation of China (Grant No. NSFC 31360591).

Availability of data and materials

We have submitted raw data through supplementary materials.

\section{Declarations}

Ethics approval and consent to participate

The sampling process did not cause any damage to the vaginal mucosa of bactrian camel. In this experiment, the breeding environment was in 
compliance with the standards relevant to an ordinary animal laboratory facility in China National Standard "Laboratory animal environment and facilities" (GB14925-2010). The feeding of and the experimental operations on animals were in accordance with the animal welfare requirements. All experimental procedures were approved by the animal protection and use committee of Inner Mongolia Agricultural University and strictly followed animal welfare and ethical guidelines.

\section{Consent for publication}

Not applicable.

\section{Competing interests}

The authors declare that they have no competing interests.

\section{Author details}

${ }^{1}$ College of Veterinary Medicine, Key Laboratory of Clinical Diagnosis and Treatment Technology in Animal Disease, Ministry of Agriculture and Rural Affairs, Inner Mongolia Agricultural University, Hohhot 010018, P.R. China. ${ }^{2}$ Veterinary Administration Bureau of Bayannur City, Linhe District, Bayannur City 015000, Inner Mongolia Autonomous Region, P.R. China. ${ }^{3}$ Alxa League Institute of Animal Health Supervision, Bayanhot 750306, Inner Mongolia Autonomous Region, P.R. China. ${ }^{4}$ Detachment of Alxa League Agriculture and Animal Husbandry Comprehensive Administrative Law Enforcement, Bayanhot 750306, Inner Mongolia Autonomous Region, P.R. China.

Received: 23 April 2021 Accepted: 23 February 2022

Published online: 05 March 2022

\section{References}

1. Ji R, Cui P, Ding F, Geng J, Gao H, Zhang H, Yu J, Hu S, Meng H. Monophyletic origin of domestic bactrian camel (Camelus bactrianus) and its evolutionary relationship with the extant wild camel (Camelus bactrianus ferus). Anim Genet. 2009;40(4):377-82.

2. Schumann H, Ribbeck R, Beulig W. Wohlfahrtia magnifica (Schiner, 1862) Diptera: Sacrophagidae) causing a vaginal myiasis in domesticated twohumped camels in the Mongolian People's republic. Arch Exp Veterinarmed. 1976;30(6):799-806.

3. Robbins K, Khachemoune A. Cutaneous myiasis: a review of the common types of myiasis. Int J Dermatol. 2010;49(10):1092-8.

4. Hadani A, Ben Yaakov B, Rosen S. Myiasis caused by Wohlfahrtia magnifica (Schiner, 1862) in the Arabian camel (Camelus dromedarius) in the Peninsula of Sinai. Rev Elev Med Vet Pays Trop. 1989;42(1):33-8.

5. Valentin A, Baumann MP, Schein E, Bajanbileg S. Genital myiasis (Wohlfahrtiosis) in camel herds of Mongolia. Vet Parasitol. 1997;73(3-4):335-46.

6. Giangaspero A, Traversa D, Trentini R, Scala A, Otranto D. Traumatic myiasis by Wohlfahrtia magnifica in Italy. Vet Parasitol. 2011;175(1-2):109-12.

7. Sazmand A, Joachim A. Parasitic diseases of camels in Iran (1931-2017) a literature review. Parasite. 2017:24:21.

8. Srinivasan M, Adnane M, Archunan G. Significance of cervico-vaginal microbes in bovine reproduction and pheromone production - a hypothetical review. Res Vet Sci. 2021;135:66-71.

9. Sroka-Oleksiak A, Gosiewski T, Pabian W, Gurgul A, Kapusta P, LudwigSłomczyńska AH, Wołkow PP, Brzychczy-Włoch M. Next-Generation Sequencing as a tool to detect vaginal microbiota disturbances during pregnancy. Microorganisms. 2020;8(11):1813.

10. Tsimaris P, Giannouli A, Tzouma C, Athanasopoulos N, Creatsas G, Deligeoroglou E. Alleviation of vulvovaginitis symptoms: can probiotics lead the treatment plan? Benef Microbes. 2019;10(8):867-72.

11 Barrientos-Durán A, Fuentes-López A, de Salazar A, Plaza-Díaz J, García F. Reviewing the composition of vaginal microbiota: inclusion of nutrition and probiotic factors in the maintenance of eubiosis. Nutrients. 2020;12(2):419.

12. Hyman RW, Fukushima M, Diamond L, Kumm J, Giudice LC, Davis RW. Microbes on the human vaginal epithelium. Proc Natl Acad Sci U S A. 2005;102(22):7952-7.

13. White BA, Creedon DJ, Nelson KE, Wilson BA. The vaginal microbiome in health and disease. Trends Endocrinol Metab. 2011;22(10):389-93.
14. Ma B, Forney LJ, Ravel J. Vaginal microbiome: rethinking health and disease. Annu Rev Microbiol. 2012;66:371-89.

15. Best AA, Porter AL, Fraley SM, Fraley GS. Characterization of gut microbiome dynamics in developing pekin ducks and impact of management system. Front Microbiol. 2016;7:2125.

16. Clemmons BA, Reese ST, Dantas FG, Franco GA, Smith TPL, Adeyosoye Ol, Pohler KG, Myer PR. Vaginal and uterine bacterial communities in postpartum lactating cows. Front Microbiol. 2017;8:1047.

17. Bokulich NA, Subramanian S, Faith JJ, Gevers D, Gordon JI, Knight R, Mills DA, Caporaso JG. Quality-filtering vastly improves diversity estimates from Illumina amplicon sequencing. Nat Methods. 2013;10(1):57-9.

18. Asnicar F, Weingart G, Tickle TL, Huttenhower C, Segata N. Compact graphical representation of phylogenetic data and metadata with GraPhIAn. PeerJ. 2015;3:e1029.

19. White JR, Nagarajan N, Pop M. Statistical methods for detecting differentially abundant features in clinical metagenomic samples. PLoS Comput Biol. 2009;5(4):e1000352.

20. Kau AL, Ahern PP, Griffin NW, Goodman AL, Gordon Jl. Human nutrition, the gut microbiome and the immune system. Nature. 2011;474(7351):327-36.

21. Rooks MG, Garrett WS. Gut microbiota, metabolites and host immunity. Nat Rev Immunol. 2016;16(6):341-52.

22. Wang J, Linnenbrink M, Künzel S, Fernandes R, Nadeau MJ, Rosenstiel P, Baines JF. Dietary history contributes to enterotype-like clustering and functional metagenomic content in the intestinal microbiome of wild mice. Proc Natl Acad Sci U S A. 2014;111(26):E2703-2710.

23. Ost KS, Round JL. Communication between the microbiota and mammalian immunity. Annu Rev Microbiol. 2018;72:399-422.

24. Hooper LV, Littman DR, Macpherson AJ. Interactions between the microbiota and the immune system. Science. 2012;336(6086):1268-73.

25. Sommer F, Bäckhed F. The gut microbiota-masters of host development and physiology. Nat Rev Microbiol. 2013;11(4):227-38.

26. Chu H, Mazmanian SK. Innate immune recognition of the microbiota promotes host-microbial symbiosis. Nat Immunol. 2013;14(7):668-75.

27. Chen YE, Fischbach MA, Belkaid Y. Skin microbiota-host interactions. Nature. 2018;553(7689):427-36.

28. Boskey ER, Telsch KM, Whaley KJ, Moench TR, Cone RA. Acid production by vaginal flora in vitro is consistent with the rate and extent of vaginal acidification. Infect Immun. 1999;67(10):5170-5.

29. Boris $S$, Barbés $C$. Role played by lactobacilli in controlling the population of vaginal pathogens. Microbes Infect. 2000;2(5):543-6.

30. Brabin L, Roberts SA, Fairbrother E, Mandal D, Higgins SP, Chandiok S, Wood P, Barnard G, Kitchener HC. Factors affecting vaginal $\mathrm{pH}$ levels among female adolescents attending genitourinary medicine clinics. Sex Transm Infect. 2005;81(6):483-7.

31. van Oostrum N, De Sutter P, Meys J, Verstraelen H. Risks associated with bacterial vaginosis in infertility patients: a systematic review and metaanalysis. Hum Reprod. 2013;28(7):1809-15.

32. Huang B, Fettweis JM, Brooks JP, Jefferson KK, Buck GA. The changing landscape of the vaginal microbiome. Clin Lab Med. 2014;34(4):747-61.

33. O'Hanlon DE, Moench TR, Cone RA. Vaginal pH and microbicidal lactic acid when lactobacilli dominate the microbiota. PLOS ONE. 2013:8(11):e80074.

34. Lamont RF, Sobel JD, Akins RA, Hassan SS, Chaiworapongsa T, Kusanovic JP, Romero R. The vaginal microbiome: new information about genital tract flora using molecular based techniques. BJOG. 2011;118(5):533-49.

35. Aldunate M, Srbinovski D, Hearps AC, Latham CF, Ramsland PA, Gugasyan R, Cone RA, Tachedjian G. Antimicrobial and immune modulatory effects of lactic acid and short chain fatty acids produced by vaginal microbiota associated with eubiosis and bacterial vaginosis. Front Physiol. 2015;6:164.

36. Antonio MA, Hawes SE, Hillier SL. The identification of vaginal Lactobacillus species and the demographic and microbiologic characteristics of women colonized by these species. J Infect Dis. 1999;180(6):1950-6.

37 Fichorova RN, Onderdonk AB, Yamamoto H, Delaney ML, DuBois AM, Allred E, Leviton A. Maternal microbe-specific modulation of inflammatory response in extremely low-gestational-age newborns. MBio. 2011;2(1):e00280-e1210.

38. Fashemi B, Delaney ML, Onderdonk AB, Fichorova RN. Effects of feminine hygiene products on the vaginal mucosal biome. Microb Ecol Health Dis. 2013;24(1):19703. 
39. Davis FM, Gallagher K. Time heals all wounds ... But wounds heal faster with lactobacillus. Cell Host Microbe. 2018;23(4):432-4.

40. MacArthur Clark JA, Sun D. Guidelines for the ethical review of laboratory animal welfare People's Republic of China National Standard GB/T 35892-2018 [lssued 6 February 2018 Effective from 1 September 2018]. Animal Model Exp Med. 2020;3(1):103-13.

\section{Publisher's Note}

Springer Nature remains neutral with regard to jurisdictional claims in published maps and institutional affiliations.

- fast, convenient online submission

- thorough peer review by experienced researchers in your field

- rapid publication on acceptance

- support for research data, including large and complex data types

- gold Open Access which fosters wider collaboration and increased citations

- maximum visibility for your research: over $100 \mathrm{M}$ website views per year

At $\mathrm{BMC}$, research is always in progress.

Learn more biomedcentral.com/submissions 